\title{
Swelling Properties of New Hydrogels Based on the Dimethyl Amino Ethyl Acrylate Methyl Chloride Quaternary Salt with Acrylic Acid and 2-Methylene Butane-1,4-Dioic Acid Monomers in Aqueous Solutions
}

\author{
Issa Katime ${ }^{1}$, Eduardo Mendizábal ${ }^{2}$ \\ ${ }^{1}$ Grupo de Nuevos Materialesy Espectroscopia Supramolecular, Departamento de Química Física, Facultad de Ciencia y Tecnología \\ (Campus de Leioa), Universidad del País Vasco, Bilbao, España; ${ }^{2}$ CUCEI, Universidad de Guadalajara, Guadalajara, México. \\ Email: issa.katime@ehu.es, lalomendizabal@gmail.com
}

Received May $22^{\text {nd }}, 2010$; revised June $17^{\text {th }}, 2010$; accepted July $21^{\text {st }}, 2010$.

\begin{abstract}
Hydrogels of dimethylaminoethyl acrylate methyl chloride quaternary salt (Q9) have been synthesized with different monomer ratio by copolymerization of this poorly studied monomer either with acrylic acid or with 2-methylene butane-1,4-dioic acid. Hydrogel swelling was measured as a function of the composition of the hydrogel and of the crosslinking agent ratio. High values of swelling have been obtained at very high crosslinking values $(<14 w t \%)$ and the equilibrium swelling was reached at very low time (less than 15 minutes). The swelling isotherms consisted of a steep initial portion and then levelled off as asymptotically to the equilibrium swelling limit. The experimental data suggest clearly that the swelling process obeys second-order kinetics. According to this, the kinetics rate constant and the equilibrium water content were determined at different comonomer composition and crosslinker concentration. The calculated kinetic constants ranged from 0.48 to $3.76 \times 10^{-2} \mathrm{~min}^{-1}$ for poly (acrylic acid-co-Q9) hydrogels and from 0.68 to $4.0 \times 10^{-2} \mathrm{~min}^{-1}$ for poly (2-methylene butane-1,4-dioic acid-co-Q9) hydrogels depending on the hydrogels composition. The diffusion process was evaluated for each hydrogel showing a non-Fickian type diffusion. In all cases was observed a considerable increase in diffusion coefficient as $Q 9$ content increases.
\end{abstract}

Keywords: Dimethyl Amino Ethyl Acrylate Methyl Chloride Quaternary Salt, 2-methylene butane-1,4-Dioic Acid, Acrylic Acid, Swelling, Diffusion Coefficients, Kinetic Order

\section{Introduction}

A gel is a polymer network which swell when immersed in solvent, but which is prevented from dissolving by the presence of crosslinks which hold the structure intact. Gels which swell in aqueous solvents are called hydrogels $[1,2]$. Equilibrium water content in hydrogels is one of their basic properties. A hydrogel with high water content is generally more advantageous for medical applications because of its permeability and biocompability [3]. Usually a large swelling is accompanied by poor mechanical properties. There are several alternatives to find a compromise between "large swelling" and "good mechanical behavior". Increasing the crosslinking agent density is a way to improve the mechanical properties but this affects adversely the swelling. Copolymerization of hydrophilic monomer (which favors swelling) with a less hydrophilic monomer results in a hydrogel with good water absorbance and improved mechanical properties in the resulting hydrogel [4].

It is now well established that some gels can reversibly swell and shrink by as much as several hundred times in response to small changes in environmental conditions such as temperature, solvent composition, $\mathrm{pH}$, ionic strength, electric field, and light. Knowledge of swelling kinetics are important for designing controlled-released devices for drugs and agriculture pesticides based on swellable polymer matrices and for predicting the release rates of the active ingredients [5].

In this paper, different hydrogels have been synthesi- 
zed copolymerizing an unusual monomer, Dimethylaminoethyl acrylate methyl chloride quaternary salt (Q9) with two other common monomers: acrylic acid (AA) and 2-methylene butane-1,4-dioic acid (IA). The large swelling of these hydrogels makes them suitable as water reservoirs in agriculture. In previous studies made in our laboratory we found a percentage degree of hydratation $\mathrm{H} \%$ 4,000 for poliQ9 with 4 wt.\% of crosslinking agent. However its soft consistence could be a handicap to manipulate these polymers. For those reasons Q9 was copolymerized with others common and less hydrophilic monomers [7], acrylic acid and methylenesuccinic, to improve its mechanical properties. The influence of the hydrogel composition and the crosslinking agent on the swelling behavior of these hydrogels is reported here. Kinetics parameters and diffusion data are also reported.

\section{Experimental Part}

Materials. Dimethylaminoethyl acrylate methyl chloride quaternary salt Q9, Merck S.A), Acrylic acid (AA, Aldrich Chemical Co.) and methylenesuccinic acid (MA, Merck), analytical purity grade, were used as monomers. Methylenesuccinic acid, or Itaconic acid is an organic compound that is one of the three acids obtained by the distillation of citric acid. Acrylic acid (AA), inhibited with $200 \mathrm{ppm}$ of hydroquinone monomethyl ether, with a reported purity of $99 \%$, was obtained from Aldrich Chemical Co. The acrylic acid monomer was purified by distillation at a reduced pressure of approximately $10 \mathrm{~mm}$ $\mathrm{Hg}$. The fraction having boiling point from $39-40^{\circ} \mathrm{C}$ was collected. N, N'-methylenbysacrylamide (Aldrich Chemical) was employed as crosslinking agent and 2,2'-azobis (2-amidinopropane) dihydrochloride, V-50 (Wako), as initiator. Distilled water was employed in all the processes. All other chemicals reagents were analytical grade. In Figure 1 the chemical structures of the reagents used in this work are shown.

Hydrogel synthesis. Hydrogels were prepared by radical copolymerization at $50^{\circ} \mathrm{C}$ of dimethylaminoethyl acrylate methyl chloride quaernary salt and acrylic acid or methylenesuccinic acid in cylindrical glass tubes. A ratio of 25/75 total monomer/water in weight was used in all cases. Comonomers ratios were varied to obtain different hydrophilic properties. The initiator and crosslinking agent concentrations were $1 \%$ with respect to the monomers in all cases. After bubbling nitrogen through the reaction mixture, thermally-initiated polymerizations were carried out for 4 hours. After reaction the hydrogels were taken from the test tubes, cut into cylindrical samples immersed in water for two weeks to remove any residual monomers and uncrosslinked polymer and then dried again to determine the conversion:

$$
\text { Structure (a) }
$$

Figure 1. Structures of the monomers and crosslinker agent: (a) Dimethylaminoethyl acrylate methyl chloride quaternary salt; (b) Acrylic acid; (c) Methylenesuccinic acid; (d) NN'-methylenbisacrylamide

$$
\text { Conversion }(\%)=\frac{\mathrm{m}_{\mathrm{g}}}{\mathrm{m}_{\mathrm{o}}} \times 100
$$

Discs $1.5 \mathrm{~mm}$ diameter were obtained from the hydrogels rods. The discs were dried to constant weight and then polished to get uniform and smooth surfaces $(\sim 1$ $\mathrm{mm}$ thickness). Their dimensions were measured with a micrometer.

Hydrogel Swelling. Dynamic swelling experiments were performed by placing the discs in distilled water at $37.0 \pm 0.1^{\circ} \mathrm{C}$ (in a thermostated bath) and measuring their weight gain as a function of time. The discs were removed from the water, dried quickly and carefully with filter paper and weighted.

The degree of swelling at different times can be calculated from the following equation:

$$
\mathrm{W}(\%)=\frac{\mathrm{m}-\mathrm{m}_{0}}{\mathrm{~m}} \times 100
$$

were $\mathrm{m}_{0}$ and $\mathrm{m}$ are the weights of the initial dry sample (xerogel) and of the hydrogel, respectively. $\mathrm{W}_{\infty}$ is the water content when the equilibrium is reached. There is another useful parameter to compare similar swellings, $\mathrm{H}(\%)$, the swelling degree, which can be expressed as:

$$
\mathrm{H}(\%)=\frac{\mathrm{m}-\mathrm{m}_{0}}{\mathrm{~m}_{0}} \times 100
$$

\section{Results and Discussion}

Influence of gel composition and crosslinking ratio.

Figure 2 shows that around 80 minutes equilibrium is reached. The studied hydrogels absorb huge amounts of 
water, especially the hydrogels with a low content of acrylic acid, as Figure 2 shows. This is a consequence of the smaller acrylic acid-water interactions compared to the Q9-water interactions. This behavior is in agreement with most of the hydrogels that have been studied $[6,7]$.

Figure 3 shows the variation of the percentage degree of hydration as a function of swelling time for hydrogels prepared from copolymers with the indicated crosslinking agent ratio. An increase in the crosslinking agent ratio had as expected a decrease in the amount of swelling. As the crosslinking agent content increases, the resultant structure of the hydrogel is less flexible, and consequently the capacity of the hydrogels to deform themselves (and consequently to swell), decreases. The decrease of swelling when the crosslinking agent increases had been extensively reported $[8,9]$.

As can be seen in Figure 4, the water content at equilibrium increases as the content of Q9 is increased, according with the introduction of more hydrophilic monomer.

Swelling Kinetics. Considering second-order kinetics the swelling rate at any time may be expressed as:

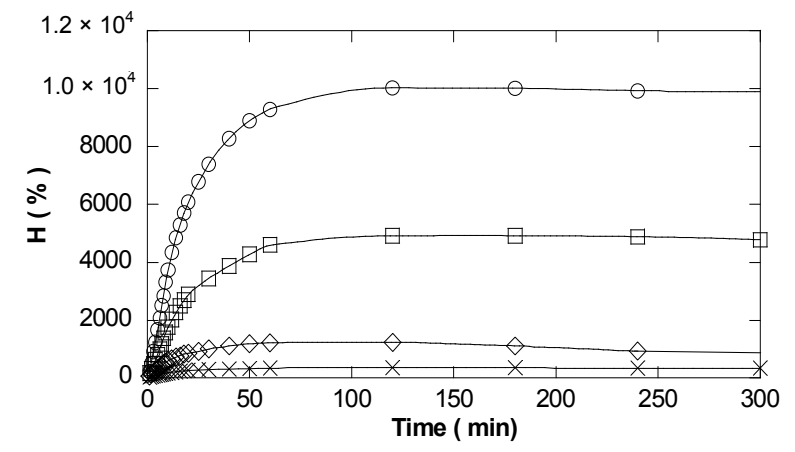

Figure 2. Swelling isotherms of hydrogels from different ratio, acrylic acid/Q9 (1 wt.\% crosslinker agent): $(\bigcirc)$ 10/90, ( $\square$ ) 20/80, (৩) 30/70, ( $\times)$ 40/60

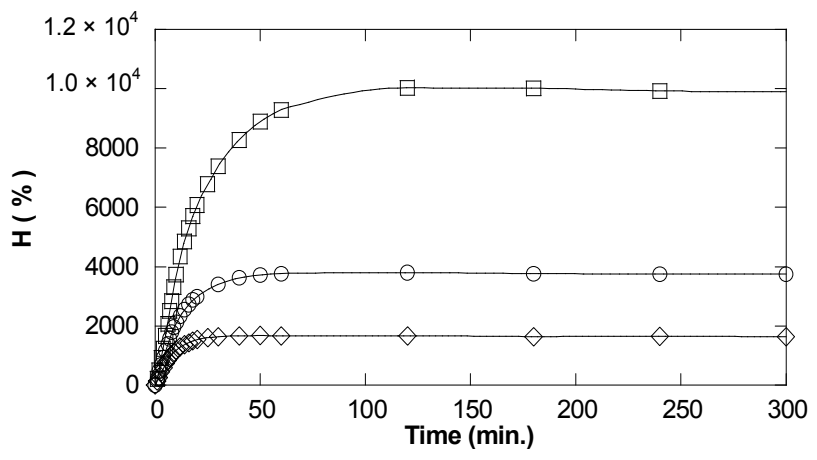

Figure 3. Swelling isotherms of 90/10 poly(acrylic acid/dimethylaminoethyl acrylate methyl chloride quaternary salt) hydrogels with different crosslinker concentration ( $\square$ ) 1 wt \%, (○) $2 \%$, (৩) 3 wt\%

$$
\frac{\mathrm{dW}}{\mathrm{dt}}=\mathrm{K}\left(\mathrm{W}_{\infty}-\mathrm{W}\right)^{2}
$$

Upon integration between the limits $(\mathrm{t}=0$ and $\mathrm{W}=0)$, the following equation is obtained,

$$
\mathrm{W}=\frac{\mathrm{KW}_{\infty}^{2} \mathrm{t}}{1+\mathrm{KW}_{\infty} \mathrm{t}}
$$

By rearranging the above equation, one gets the following expression,

$$
\frac{\mathrm{t}}{\mathrm{W}}=\frac{1}{\mathrm{KW}_{\infty}^{2}}+\frac{\mathrm{t}}{\mathrm{W}_{\infty}}
$$

According to this equation, the experimental data must fit a straight line with a slope of $1 / \mathrm{W}_{\infty}$ with ordinate of $1 / \mathrm{KW}_{\infty}^{2}$. Therefore, we can calculate the water content at equilibrium $\left(\mathrm{W}_{\infty}\right)$ and the kinetics rate constants $(\mathrm{K})$ from a plot of $t / W$ against time. The variation of $t / W$ against time is plotted in Figure 5 for the 90/10 hydrogel (Q9/ acrylic acid). In all cases the experimental data fit Equation (6) with $r=0.9995$. Similar results were obtained for

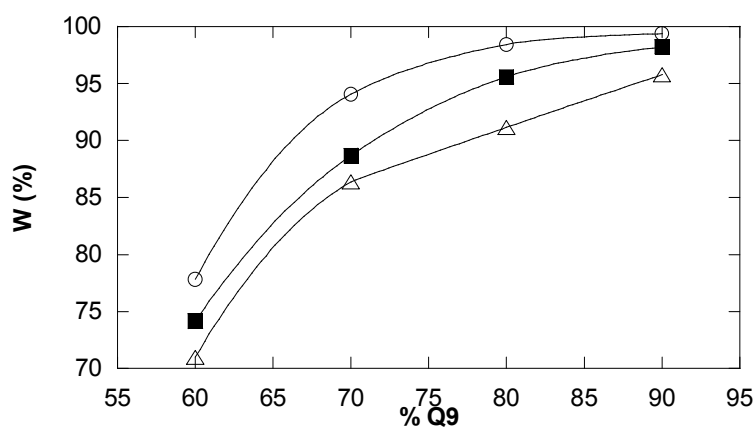

Figure 4. Variation of the water content at equilibrium, $W \infty$, as a function of $\mathrm{Q9}$ content at $37^{\circ} \mathrm{C}$ for hydrogels with different crosslinker concentration: $(\bigcirc) 1 \mathrm{wt} \%,(\square) 2 \mathrm{wt} \%,(\diamond)$ $5 \mathrm{wt} \%$

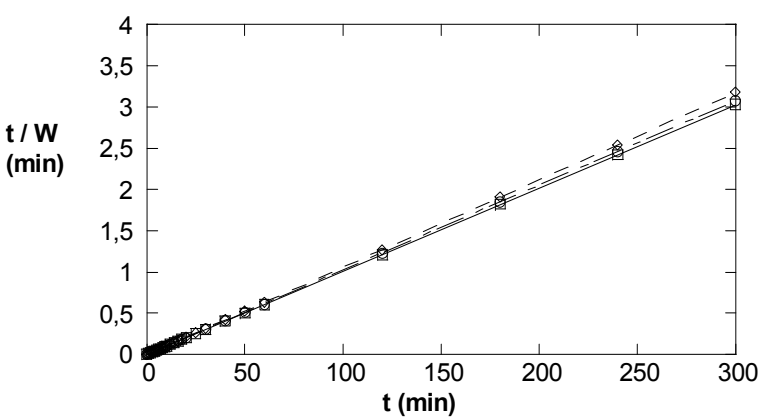

Figure 5. Experimental data of water content $W$ and time $t$ plotted according to Equation (1) (second order-kinetic) for 90/10 Q9/acrylic acid hydrogels from different crosslinking agent ratio ( $\square) 1$ wt. \%, (○) 2 wt. \%, ( $) 3$ wt. \% 
the other hydrogels. Therefore, we could calculate the wa ter content at equilibrium $\left(\mathrm{W}_{\infty}\right)$ and the kinetics rate constants $(\mathrm{K})$.

In Table 1 are summarized the experimental values of swelling kinetic rate constants $(\mathrm{K})$ as a function of the Q9 and crosslinking percentage. An increase in the crosslinking and a decrease in the more hydrophilic monomer content, drive to a higher physical restriction in the network, which causes that the swelling rate decreases.

Diffusion. When a glassy hydrogel is brought into contact with water, water diffuses into the hydrogel and the hydrogel swells [10]. Diffusion involves migration of water into pre-existing or dynamically formed spaces between hydrogel chains whereas swelling of the hydrogel involves a larger scale segmental motion resulting in a bigger separation between hydrogel chains [11]. The following useful equation was used to determinate the nature of diffusion of water into hydrogels:

$$
\frac{\mathrm{M}_{\mathrm{t}}}{\mathrm{M}_{\infty}}=\mathrm{K}^{\mathrm{n}}
$$

where $M_{t}$ and $M_{\infty}$ denote the amount of solvent diffused into the gel at time $\mathrm{t}$ and at infinite time respectively, $\mathrm{K}$ is a constant related to the structure of the network and $n$ is a characteristic exponent of the transport mode of the solvent [12]. Depending on the relative rates of diffusion and polymer relaxation three classes of diffusion mechanisms are distinguished [13]: 1) Case I or Fickian diffusion in which the rate of diffusion is much less than that of relaxation $(\mathrm{n}=0.50), 2)$ Case II diffusion, in which diffusion is very rapid compared with the relaxation processes $(n=1)$, and 3) Non-Fickian or anomalous diffusion which occurs when the diffusion and relaxation rates are comparables $(0.50<\mathrm{n}<1)$.

To elucidate the transport mechanisms, the swelling curves were fitted to the following equation:

$$
\log \left(\frac{\mathrm{M}_{\mathrm{t}}}{\mathrm{M}_{\infty}}\right)=\log \mathrm{k}+\mathrm{n} \log \mathrm{t}
$$

The exponents $\mathrm{n}$ were calculated from the slopes and values ranging from 0.66 to 1.24 were obtained (Table 2). This indicates that diffusion of water to the interior of the discs follows an anomalous diffusion mechanism, revealing the existence of certain coupling between molecular

Table 1. Experimental values of kinetics rate constant (K) as function of Q9 concentration and crosslinker concentration for poly(acrylic acid-co-Q9) hydrogels

\begin{tabular}{ccccc}
\hline $\mathbf{K} \times \mathbf{1 0}^{\mathbf{2}}\left(\mathbf{m i n}^{-1}\right)$ & \multicolumn{4}{c}{$\mathbf{( \% )} \mathbf{Q 9}$} \\
\hline $\mathbf{( \% )} \mathbf{N M B A}$ & $\mathbf{6 0}$ & $\mathbf{7 0}$ & $\mathbf{8 0}$ & $\mathbf{9 0}$ \\
\hline $\mathbf{1}$ & 0.70 & 2.00 & 2.37 & 3.76 \\
$\mathbf{2}$ & 0.56 & 1.67 & 2.19 & 3.40 \\
$\mathbf{5}$ & 0.48 & 1.43 & 1.65 & 2.47 \\
\hline
\end{tabular}

diffusion and tension relaxation which are developed during swelling of the xerogels. As Table 2 shows the hydrogel which swells the most is an unusual system with a characteristic $\mathrm{n}$ exponent higher than 1 . This table also shows that as the Q9 percentage and crosslinker concentration decreases the system deviates more from a Fickian behavior. For an anomalous mechanism the coupling between molecular transport and stress relaxation during swelling causes deviations with respect to Fickian mechanism. This becomes more important when Q9 content increases because it is a more hydrophilic monomer, and when the crosslinker increases due to the decrease in free volume.

The diffusion coefficients for non-Fickian sorption processes can be obtained by mean of the following equation [14]:

$$
D=\frac{0.049}{\left(t / 4 \ell^{2}\right)}
$$

where $t$ is the time at which the swelling is one half the equilibrium value and $\ell$ is the radius of the cylindrical hydrogel at this time. Figure 6 shows the variation of the water diffusion coefficient of water $\mathrm{i}, \mathrm{D}$, for different hydrogel compositions. Experimental values show that when the Q9 content increases, the diffusion coefficient of hydrogels, D, increases. The reason, is the higher swelling degree at equilibrium when the more hydrophilic component increases.

Hydrogels of methylenesuccinic acid/Q9. Swelling of hydrogels of methylenesuccinic acid and Q9 were also

Table 2. Values of $\mathbf{n}$ (diffusion exponent), for poly(acrylic acid-co-Q9) hydrogels as a function of Q9 composition and crosslinker concentration

\begin{tabular}{ccccc}
\hline $\mathbf{n}$ & \multicolumn{4}{c}{$\% \mathbf{Q 9}$} \\
\hline \% NMBA & $\mathbf{6 0}$ & $\mathbf{7 0}$ & $\mathbf{8 0}$ & $\mathbf{9 0}$ \\
\hline $\mathbf{1}$ & 0.87 & 0.92 & 1.00 & 1.24 \\
$\mathbf{2}$ & 0.78 & 0.80 & 0.93 & 0.97 \\
$\mathbf{5}$ & 0.66 & 0.71 & 0.77 & 0.94 \\
\hline
\end{tabular}

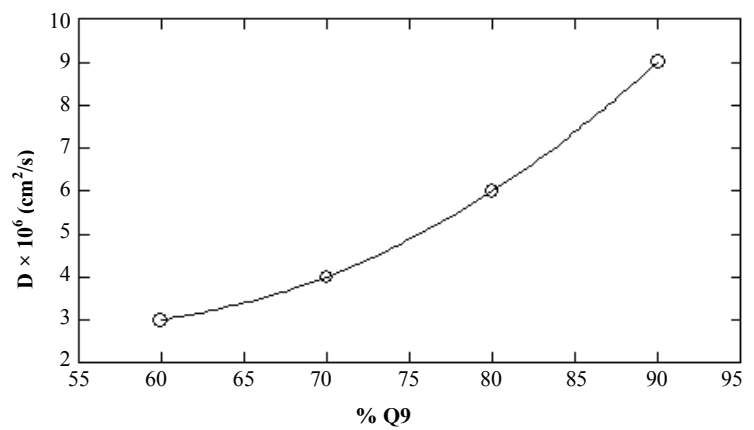

Figure 6. Variation of water diffusion coefficient at $37^{\circ} \mathrm{C}, \mathrm{D}$, of poly(acrylic acid-co-Q9) hydrogels 
studied. Figures $\mathbf{7}$ and $\mathbf{8}$ show the variation of $\% \mathrm{H}$ as a function of swelling time for hydrogels prepared with different Q9 concentration and crosslinker agent, respectively. These hydrogels show a similar swelling dependence on the content of the more hydrophilic monomer (Q9) and on crosslinker agent, than the poly(acrylic acid/Q9) hydrogels, that is a larger swelling degree is obtained by using a larger concentration of Q9 and a lower concentration of crosslinker.

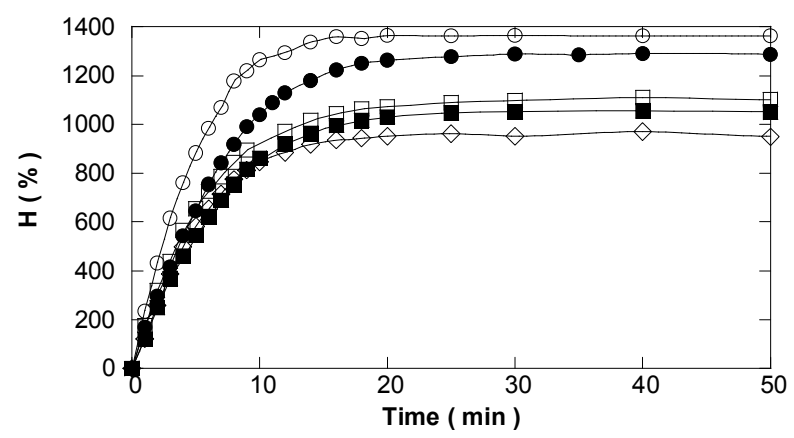

Figure 7. Swelling isotherms of methylenesuccinic acid/Q9 hydrogels $(6 \%$ crosslinker agent) for different $\mathrm{Q9}$ concentration: $(\diamond) 80 \%,(\square) 85 \%,(\square) 90 \mathrm{wt} \%,(\bullet) 95 \mathrm{wt} \%$ and $(\bigcirc)$ $100 \mathrm{wt} \%$

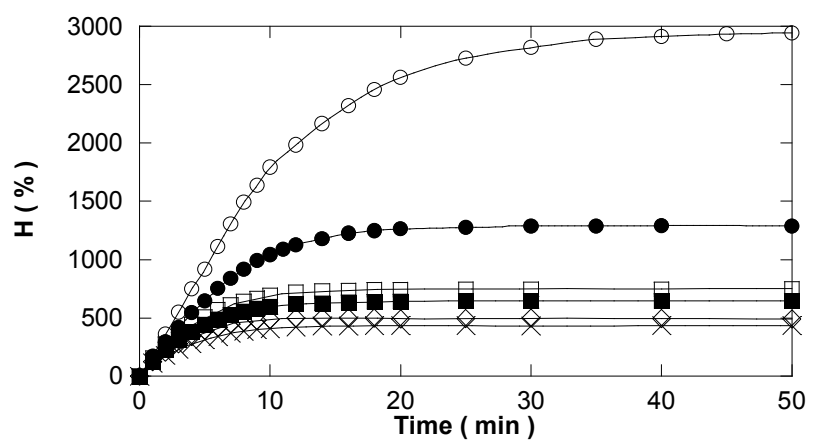

Figure 8. Swelling isotherms of 5/95 methylenesuccinic acid/Q9 hydrogels with different crosslinker concentration:

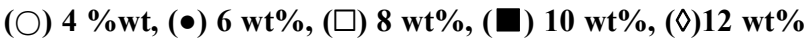
and $(X) 14 \mathrm{wt} \%$

Table 3. Kinetics rate constant of poly(methylenesuccinic acid-co-Q9) hydrogels

\begin{tabular}{ccccccc}
\hline $\mathbf{K} \times \mathbf{1 0}^{\mathbf{2}} \mathbf{~ m i n}^{-\mathbf{1}}$ & \multicolumn{7}{c}{ \% NMBA } \\
\hline $\mathbf{\%} \mathbf{Q 9}$ & $\mathbf{4}$ & $\mathbf{6}$ & $\mathbf{8}$ & $\mathbf{1 0}$ & $\mathbf{1 2}$ & $\mathbf{1 4}$ \\
\hline $\mathbf{8 0}$ & 0.68 & 2.83 & 2.30 & 3.26 & 2.79 & 4.00 \\
$\mathbf{8 5}$ & 2.14 & 2.06 & 1.92 & 3.72 & 3.65 & 3.46 \\
$\mathbf{9 0}$ & 2.75 & 2.56 & 2.32 & 2.26 & 2.21 & 2.17 \\
$\mathbf{9 5}$ & 2.61 & 2.46 & 2.33 & 2.25 & 2.06 & 2.12 \\
$\mathbf{1 0 0}$ & 2.90 & 2.89 & 2.38 & 2.62 & 2.17 & 2.46 \\
\hline
\end{tabular}

Table 4. Values of $n$ (diffusion exponent) for poly (methylenesuccinic acid-co-Q9) hydrogels as a function of Q9 composition and crosslinker concentration

\begin{tabular}{ccccccc}
\hline \multicolumn{7}{c}{ \% NMBA } \\
\hline \% Q9 & $\mathbf{4}$ & $\mathbf{6}$ & $\mathbf{8}$ & $\mathbf{1 0}$ & $\mathbf{1 2}$ & $\mathbf{1 4}$ \\
\hline $\mathbf{8 0}$ & 0.97 & 0.73 & 0.82 & 0.63 & 0.56 & 0.54 \\
$\mathbf{8 5}$ & 0.90 & 0.82 & 0.66 & 0.68 & 0.63 & 0.59 \\
$\mathbf{9 0}$ & 0.88 & 0.74 & 0.84 & 0.66 & 0.67 & 0.68 \\
$\mathbf{9 5}$ & 0.87 & 0.86 & 0.71 & 0.77 & 0.58 & 0.84 \\
$\mathbf{1 0 0}$ & 0.97 & 0.86 & 0.67 & 0.73 & 0.83 & 0.93 \\
\hline
\end{tabular}

By graphing the experimental data (not shown) using Equation (3), it was found that the swelling process of these hydrogels also obeys second-order kinetics. In Table 3 are summarized the experimental values of kinetics rate constants $(\mathrm{K})$ as a function of $\mathrm{Q} 9$ and crosslinker agent content. An increase in the crosslinking ratio and a decrease in the more hydrophilic monomer content. The values of $n$ exponent are listed in Table 4. In all cases $n$ exponent is higher than 0.50 . Therefore the water diffusion into hydrogels displays also a non-Fickian character.

\section{Conclusions}

In this paper we have described the swelling kinetics of poly(acrylic acid/dimethylaminoethyl acrylate methyl chloride quaternary salt) and poly(methylenesuccinic acid/ dimethylaminoethyl acrylate methyl chloride quaternary salt) hydrogels. These hydrogels absorb huge amounts of water. The experimental data indicate that the swelling process follows second-order kinetics for all the studied systems. Hydrogels show large swelling properties as the Q9 content increases and crosslinking agent ratio decreases.

Water diffusion into hydrogels is a non-Fickian type diffusion. Diffusion coefficients increase with increasing Q9 content .

The diffusion coefficients were determined by using equation (9). In these copolymers is observed that the crosslinking ratio has not an important effect on the water transport, as we concluded with poly(acrylic acid-codimethylaminoethyl acrylate methyl chloride quaternary) hydrogels. For lower Q9 content hydrogels, the diffusion coefficient remains constant with the composition, and in these cases the determined value was $6.5 \times 10^{-6} \mathrm{~cm}^{2} / \mathrm{s}$. It was only observed a considerable effect on diffusion coefficient due to Q9 content on 0/100 hydrogel (itaconic acid/Q9). The diffusion coefficient determined in this case was $1.4 \times 10^{-5} \mathrm{~cm}^{2} / \mathrm{s}$.

\section{Acknowledgements}

The authors thanks the financial support for this work was provided by Ministerio de Ciencia e Innovación (Pr- 
oject Number: EUI2008-00178) and the Gobierno Vasco for their financial support

\section{REFERENCES}

[1] L. W. FU and S. Chich-Hsuan, "PH-Thermoreversible Hydrogels. II. Synthesis and Swelling Behaviors of NIsopropylacrylamide-Co-Acrylic Acid-Co-Sodium Acrylate Hydrogels," Journal of Applied Polymer Science, Vol. 73, No. 10, September 1999, pp. 1955-1967.

[2] I. P. Lee, "Kinetics of Drug Release from Hydrogel Matrices," Journal of Controlled Release, Vol. 2, November 1985, pp. 277-288.

[3] I. Katime, R. Novoa and F. Zuluaga, "Swelling Kinetics and Release Studies of Theophylline and Aminophylline from Acrylic Acid/N-Alkyl Methacrylate Hydrogels," European Polymer Journal, Vol. 37, No. 7, July 2001, pp. $1465-1471$

[4] A. Nagaoka, "Mechanical Properties of Composite Hydrogels," Polymer Journal, Vol. 21, No. 10, April 2005, pp. 847-850.

[5] H. Schott, "Swelling Kinetics of Polymers," Journal of Macromolecular Science, Vol. 31, No. 1, March 1992, pp. $1-9$.

[6] N. M. Franson and N. A. Pepas, "Influence of Copolymer Composition on Non-Fickian Water Transport through Glassy Copolymers," Journal of Applied Polymer Science, Vol. 28, No. 4, April 1983, pp. 1299-1310.

[7] I. Katime and E. Rodríguez, "Synthesis and Swelling
Kinetics of Poly(Acrylic Acid-Co-Itaconic Acid) Hydrogels," Recent Research Developments in Polymer Science, Vol. 5, 2001, pp. 139-152.

[8] P. E. M. Allen, D. J. Bennette and D. R. G. Williams, "Water in Methacrylates-I. Sorption and Desorption Properties of Poly(2-Hydroxyethyl Methacrylate-Co-Glycol Dimethacrylate) Networks," European Polymer Journal, Vol. 28, No. 4, April 1992, pp. 347-352.

[9] C. S. Brazel and N. A. Pepas, "Mechanisms of solute and Drug Transport in Relaxing, Swellable, Hydrophilic Glassy Polymers," Polymer, Vol. 40, No. 12, June 1999, pp. 3383-3398

[10] I. Katime, N. Valderruten and J. R. Quintana, "Controlled Release of Aminophylline from Poly (N-IsopropylacrylamideCo-Itaconic Acid) Hydrogels," Polymer International, Vol. 50, No. 8, August 2001, pp. 869-879.

[11] A. R. Berens and H. B. Hopfenberg, "Diffusion and Relaxation in Glassy Polymer Powders: 2. Separation of Diffusion and Relaxation Parameters," Polymer, Vol. 19, No. 5, May 1978, pp. 489-496.

[12] H. L. Frisch, "Sorption and Transport in Glassy Polymers-A Review," Polymer Engineering \& Science, Vol. 20, No. 1, January 1980, pp. 2-13.

[13] J. Crank "The Mathematics of Diffusion," Clarendon Press, Oxford, 1975.

[14] I. Katime, R. Novoa, E. Díaz de Apodaca, E. Mendizábal and J. E. Puig, "Theophylline Release from Poly(Acrylic Acid-Co-Acrylamide) Hydrogels," Polymer Testing, Vol. 18, No. 7, October 1999, pp. 559-566. 